\title{
A New Approach to Calorimetry in Space-Based Experiments for High-Energy Cosmic Rays ${ }^{\dagger}$
}

Gabriele Bigongiari ${ }^{1,2}, * \oplus$, Oscar Adriani ${ }^{3,4} \oplus$, Sebastiano Albergo ${ }^{5,6}$, Giovanni Ambrosi ${ }^{7}$, Lucrezia Auditore ${ }^{6,8}$, Andrea Basti ${ }^{1}$, Eugenio Berti ${ }^{3,4}$, Lorenzo Bonechi ${ }^{4}$, Simone Bonechi ${ }^{1,2}$, Massimo Bongi ${ }^{3,4}$, Valter Bonvicini ${ }^{9}$, Sergio Bottai ${ }^{4}$, Paolo Brogi ${ }^{1,2}$, Gigi Cappello ${ }^{6}$, Paolo Walter Cattaneo ${ }^{10}$, Raffaello D'Alessandro ${ }^{3,4}$, Sebastiano Detti ${ }^{4}$, Matteo Duranti ${ }^{7}$, Mauro Fasoli ${ }^{11,12}$, Noemi Finetti ${ }^{4,13}{ }^{10}$, Valerio Formato ${ }^{7}$, Maria Ionica ${ }^{7}$, Antonio Italiano ${ }^{6}$, Piergiulio Lenzi ${ }^{3,4}$, Paolo Maestro ${ }^{1,2}$, Pier Simone Marrocchesi ${ }^{1,2}$, Nicola Mori ${ }^{4}$, Giulio Orzan ${ }^{9}$, Miriam Olmi ${ }^{3,4}$, Lorenzo Pacini ${ }^{4,14}$, Paolo Papini ${ }^{4}$, Maria Grazia Pellegriti ${ }^{6}$, Andrea Rappoldi ${ }^{10}$, Sergio Bruno Ricciarini ${ }^{4,14}$, Antonella Sciuto ${ }^{6,15}$, Gianluigi Silvestre ${ }^{7,16}$,

Oleksandr Starodubtsev ${ }^{4}$, Francesco Stolzi ${ }^{1,2}$, Jung Eun Suh ${ }^{1,2}$, Arta Sulaj ${ }^{1,2}$, Alessio Tiberio ${ }^{3,4}$, Alessia Tricomi 5,6, Antonio Trifirò 6,17, Marina Trimarchi 6,17, Elena Vannuccini ${ }^{4}$, Anna Vedda ${ }^{11,12}$, Gianluigi Zampa ${ }^{9}$ and Nicola Zampa ${ }^{9}$

1 INFN Pisa, Largo Bruno Pontecorvo 3, I-56127 Pisa, Italy; andrea.basti@pi.infn.it (A.B.); bonechi2@unisi.it (S.B.); paolo.brogi@unisi.it (P.B.); paolo.maestro@pi.infn.it (P.M.); piersimone.marrocchesi@pi.infn.it (P.S.M.); francesco.stolzi@unisi.it (F.S.); jungeun.suh@pi.infn.it (J.E.S.); arta.sulaj@pi.infn.it (A.S.)

2 Dipartimento di Scienze Fisiche, della Terra e dell'Ambiente, Università di Siena, Strada Laterina 8, I-53100 Siena, Italy

3 Dipartimento di Fisica e Astronomia, Università di Firenze, via G. Sansone 1, I-50019 Sesto Fiorentino (Firenze), Italy; adriani@fi.infn.it (O.A.); eugenio.berti@fi.infn.it (E.B.); massimo.bongi@fi.infn.it (M.B.); candi@fi.infn.it (R.D.A.); piergiulio.lenzi@cern.ch (P.L.); miriam.olmi@gssi.it (M.O.); tiberio@fi.infn.it (A.T.)

4 INFN Firenze, via B. Rossi 1, I-50019 Sesto Fiorentino (Firenze), Italy; lorenzo.bonechi@fi.infn.it (L.B.); bottai@fi.infn.it (S.B.); sebastiano.detti@fi.infn.it (S.D.); noemi.finetti@cc.univaq.it (N.F.); mori@fi.infn.it (N.M.); 1.pacini@ifac.cnr.it (L.P.); papini@fi.infn.it (P.P.); s.ricciarini@ifac.cnr.it (S.B.R.); oleksandr.starodubtsev@fi.infn.it (O.S.); vannuccini@fi.infn.it (E.V.)

5 Dipartimento di Fisica e Astronomia, Università di Catania, via S. Sofia 74, I-95123 Catania, Italy; sebastiano.albergo@ct.infn.it (S.A.); alessia.tricomi@ct.infn.it (A.T.)

6 INFN Catania, via S. Sofia 64, I-95123 Catania, Italy; lauditore@unime.it (L.A.); gigi.cappello@ct.infn.it (G.C.); antonio.italiano@ct.infn.it (A.I.); mariagrazia.pellegriti@ct.infn.it (M.G.P.); antonella.sciuto@imm.cnr.it (A.S.); antonio.trifiro@unime.it (A.T.); mtrimarchi@unime.it (M.T.)

7 INFN Perugia, via A. Pascoli, I-06100 Perugia, Italy; Giovanni.Ambrosi@pg.infn.it (G.A.); matteo.duranti@pg.infn.it (M.D.); valerio.formato@pg.infn.it (V.F.); maria.ionica@pg.infn.it (M.I.); gianluigi.silvestre@pg.infn.it (G.S.)

8 Dipartimento di Scienze Biomediche, Odontoiatriche e delle Immagini Morfologiche e Funzionali Universita' di Messina, sal. Sperone 31, I-98121 Messina, Italy

9 INFN Trieste, via Valerio 2, I-34127 Trieste, Italy; bonvicini@ts.infn.it (V.B.); giulio.orzan@ts.infn.it (G.O.); gianluigi.zampa@ts.infn.it (G.Z.); Nicola.Zampa@ts.infn.it (N.Z.)

10 INFN Pavia, via A. Bassi 6, I-27100 Pavia, Italy; Paolo.Cattaneo@pv.infn.it (P.W.C.); Andrea.Rappoldi@pv.infn.it (A.R.)

11 Dipartimento di Scienza dei Materiali, Università di Milano-Bicocca, via Cozzi 55, I-20125 Milano, Italy; mauro.fasoli@mater.unimib.it (M.F.); anna.vedda@unimib.it (A.V.)

12 INFN Milano-Bicocca, Piazza della Scienza, 3-20154 Milano, Italy

13 Dipartimento di Scienze Fisiche e Chimiche, Università dell'Aquila, Via Vetoio, Coppito, 67100 L'Aquila, Italy

14 IFAC (CNR), via Madonna del Piano 10, I-50019 Sesto Fiorentino (Firenze), Italy

15 CNR IMM Catania, Ottava strada, 5-95121 Catania, Italy

16 Dipartimento di Fisica e Geologia, Università di Perugia, via A. Pascoli, I-06100 Perugia, Italy 
17 Dipartimento di Scienze Matematiche e Informatiche, Scienze Fisiche e Scienze della Terra, Università di Messina, sal. Sperone 31, I-98166 Messina, Italy

* Correspondence: gabriele.bigongiari@pi.infn.it; Tel.: +39-050-2214-349

+ This paper is based on the talk at the 7th International Conference on New Frontiers in Physics (ICNFP 2018), Crete, Greece, 4-12 July 2018.

Received: 28 November 2018; Accepted: 4 February 2019; Published: 7 March 2019

\begin{abstract}
Precise measurements of the energy spectra and of the composition of cosmic rays in the PeV region could improve our knowledge regarding their origin, acceleration mechanism, propagation, and composition. At the present time, spectral measurements in this region are mainly derived from data collected by ground-based detectors, because of the very low particle rates at these energies. Unfortunately, these results are affected by the high uncertainties typical of indirect measurements, which depend on the complicated modeling of the interaction of the primary particle with the atmosphere. A space experiment dedicated to measurements in this energy region has to achieve a balance between the requirements of lightness and compactness, with that of a large acceptance to cope with the low particle rates. CaloCube is a four-year-old R\&D project, approved and financed by the Istituto Nazionale di Fisica Nucleare (INFN) in 2014, aiming to optimize the design of a space-borne calorimeter. The large acceptance needed is obtained by maximizing the number of entrance windows, while thanks to its homogeneity and high segmentation this new detector achieves an excellent energy resolution and an enhanced separation power between hadrons and electrons. In order to optimize detector performances with respect to the total mass of the apparatus, comparative studies on different scintillating materials, different sizes of crystals, and different spacings among them have been performed making use of MonteCarlo simulations. In parallel to simulations studies, several prototypes instrumented with CsI(Tl) (Caesium Iodide, Tallium doped) cubic crystals have been constructed and tested with particle beams. Moreover, the last development of CaloCube, the Tracker-In-Calorimeter (TIC) project, financed by the INFN in 2018, is focused on the feasibility of including several silicon layers at different depths in the calorimeter in order to reconstruct the particle direction. In fact, an important requirement for $\gamma$-ray astronomy is to have a good angular resolution in order to allow precise identification of astrophysical sources in space. In respect to the traditional approach of using a tracker with passive material in front of the calorimeter, the TIC solution can save a significant amount of mass budget in a space satellite experiment, which can then be exploited to improve the acceptance and the resolution of the calorimeter. In this paper, the status of the project and perspectives for future developments are presented.
\end{abstract}

Keywords: cosmic rays; astroparticles; $\gamma$-ray astronomy

\title{
1. Introduction
}

The direct measurement of cosmic-ray (CR) spectra in the $\mathrm{PeV}$ region will be the instrumental challenge for future CR experiments. Indirect measurements on the ground identified, around this energy region, a sudden steeping in the inclusive spectrum of particles, a feature known as the CR "knee", and a progressively heavier composition. Therefore, the precise knowledge of particle spectra and composition in this spectral region could allow important discoveries in the field of high-energy $\mathrm{CR}$ physics. Direct CR detection permits a univocal elemental identification and a more precise energy measurement but suffers from low exposure due to low CR rates. Currently the DArk Matter Particle Explorer (DAMPE) [1], the largest experiment operating in space, is expected to measure the flux of electrons+positrons up to $\sim 10 \mathrm{TeV}$ and the fluxes of protons and nuclei up to $\sim 100 \mathrm{TeV}$ per nucleon. Future space satellite experiments must be carefully designed in order to extend these measurements 
of about one order of magnitude in energy (up to some tens of $\mathrm{TeV}$ for electrons+positrons, up to some units of $\mathrm{PeV}$ per nucleon for protons and nuclei). In particular, assuming five years of operations, they must have a good energy resolution $\sigma_{E} / E(<2 \%$ for electromagnetic showers, $<40 \%$ for hadronic showers) and a good effective geometric factor $G_{e f f}\left(>3.5 \mathrm{~m}^{2} \mathrm{sr}\right.$ for electromagnetic showers, $>2.5 \mathrm{~m}^{2} \mathrm{sr}$ for hadronic showers), where the effective geometric factor is defined as the product between the geometric factor $\mathrm{G}$ and the analysis selection efficiency $\epsilon$ (hence it depends both on the design geometry and the shower containment of the detector).

\section{The Basic Idea}

The major constraint, in achieving the performances discussed above, is the limitation in weight for these kinds of detectors (a few tons), which severely affects both the geometrical factors and the energy resolution. The aim of the CaloCube $R \& D$ project is the design optimization of a space-borne calorimeter to extend the range of direct $\mathrm{CR}$ measurements up to the $\mathrm{PeV}$ region in order to measure the "knee" of the lightest components [2]. The basic idea is to develop and optimize a cubic calorimeter made of $\mathrm{N} \times \mathrm{N} \times \mathrm{N}$ scintillating crystals, each one having a cubic geometry of 1 Molière radius $\left(R_{M}\right)$ per side and being equipped with a system of two photodiodes (PDs).For example, assuming a CsI(Tl) calorimeter of 2 tons (including both crystals and support), each scintillator has a $3.6 \mathrm{~cm}$ side with a gap between scintillators of $0.3 \mathrm{~cm}$, for a total number of crystals of $20 \times 20 \times 20$. In this way, the total depth of the calorimeter is $78 \mathrm{~cm}$, corresponding to 39 radiation lengths $\left(X_{0}\right)$ and 1.9 nuclear interaction lengths $\left(\lambda_{I}\right)$ for vertical particles. This design, schematically shown in Figure 1, has three main advantages. First of all, being as homogeneous as possible, it has a good energy resolution, especially in the case of electromagnetic showers. Secondly, being as isotropic as possible, it has a good geometric factor because, assuming the employment of a face for mechanical support, the cubic geometry provides the possibility to collect particles from either the top or the lateral faces without any difference in reconstruction performances: in the case of a $\mathrm{CsI}(\mathrm{Tl})$ calorimeter, this solution increases the geometrical factor from $1.91 \mathrm{~m}^{2} \mathrm{sr}$ (single face) to $9.55 \mathrm{~m}^{2} \mathrm{sr}$ (five faces). Lastly, the fine segmentation allows us to reconstruct the 3D profile of the shower, useful for energy reconstruction and for electron-to-proton discrimination [3]. In order to measure an energy deposit going from the Minimum Ionizing Particle (MIP) signal necessary for channels calibration $(\sim 10 \mathrm{MeV})$ to the one released in a single crystal from a shower of a proton near the knee region $(\sim 100 \mathrm{TeV})$, a very large $\left(\sim 10^{7}\right)$ dynamic range is required. This is achieved by employing a simple and compact readout scheme obtained by equipping each crystal with two photodiodes (PDs): a large area PD for small signals and a small area PD for large signals.
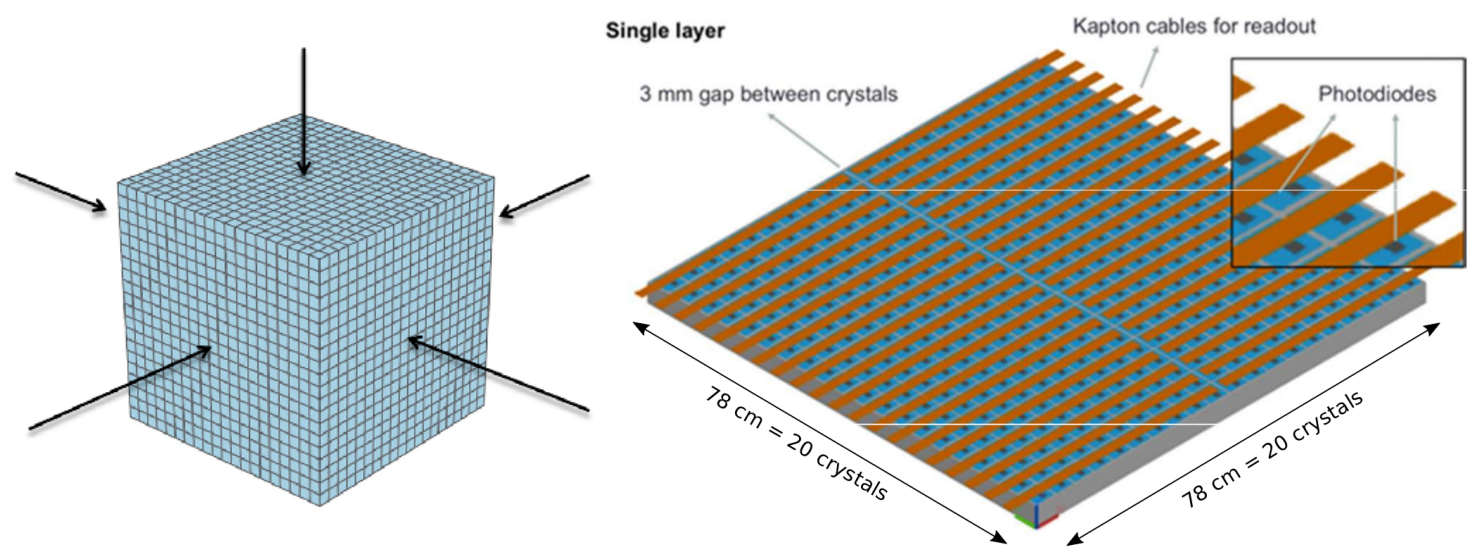

Figure 1. Conceptual design of the CaloCube 3D highly-segmented calorimeter: On the left, the complete cubic detector; on the right, one of the 20 layers. 


\section{Monte Carlo Studies and Expected Performances}

A model of the calorimeter, based on the FLUKA package [4], was simulated, in order to evaluate the performances and to optimize the design. A comparative study of different scintillating materials was done, among them $\mathrm{CsI}(\mathrm{Tl}), \mathrm{BaF} 2, \mathrm{YAP}(\mathrm{Yb}), \mathrm{BGO}$, and $\mathrm{LYSO}(\mathrm{Ce})$ crystals. For hadron detection, the best choice is a compromise between size (density of the absorber) and shower containment (interaction length), which determine energy resolution. The geometric parameters were defined by assuming about 2 tons of active material in total; the size of the single cube was fixed to $1 R_{M}$ and the gap among adjacent elements was rescaled to obtain the same active volume fraction $(\sim 78 \%)$. The signal induced in the PDs by the scintillation light was evaluated by accounting for the light yield of the scintillators, the light collection efficiency on one face, the size, and the quantum efficiency of the PD at the emission peak. Direct ionization on the PD was also considered.

The performances of the detector in terms of $\sigma_{E} / E$ and $G_{\text {eff }}$ were then investigated injecting an isotropic flux of $100-1000 \mathrm{GeV}$ electrons, $1-1000 \mathrm{TeV}$ protons, and $1 \mathrm{TeV}$ nuclei on a single face. The results are shown in left panel of Figure 2 in the case of $1 \mathrm{TeV}$ protons, where we can see that all the scintillators satisfy the requirements defined in the previous section, but the best ones are the crystals having a low value of $\lambda_{I}$. This is because, even if the size of the calorimeter is small as in this case, the better shower containment leads to a larger $G_{e f f}$, as well as to a better $\sigma_{E} / E$. Among the five materials, LYSO is the best candidate for future space satellite experiments, leading to $\sigma_{E} / E \sim 32.5 \%$ and $G_{e f f} \sim$ $4 \mathrm{~m}^{2} \mathrm{sr}$. The high energy cosmic-radiation detection (HERD) facility is a scientific experiment on-board China's Space Station, planned for operation starting around 2025 and lasting for about 10 years, with a design inspired by the CaloCube idea. The main instrument of HERD is a 3D calorimeter made of about 7500 cubes of LYSO crystals, corresponding to about 55 radiation lengths and 3 nuclear interaction lengths, respectively [5]. The performances of the calorimeter for electrons in the energy range between 100 and $1000 \mathrm{GeV}$ were also investigated. As depicted in the right panel of Figure 2, in this case $\sigma_{E} / E$ and $G_{e f f}$ are $2 \%$ and $3.4 \mathrm{~m}^{2}$ sr, respectively, if we select only events with a shower length longer than $22 X_{0}$. Further details about the MonteCarlo studies can be found in Reference [6].
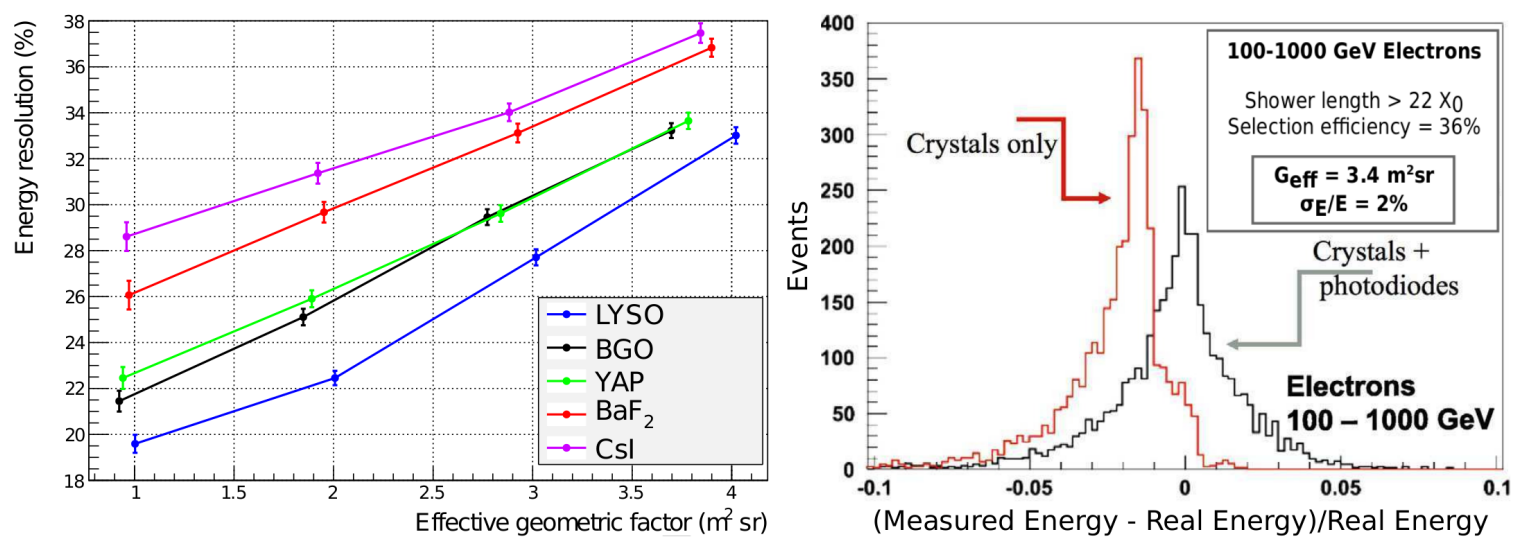

Figure 2. Left panel: Average energy resolution as a function of the effective geometric factor, obtained by applying a progressively looser cut on the shower length in the case of $1 \mathrm{TeV}$ protons; Right panel: energy resolution of the detector for 100-1000 GeV incident electrons, in case of the energy deposit in crystals only (red) or in both crystals and photodiodes (black).

\section{The Prototype}

Starting from 2012, several prototypes of the CaloCube detector have been built [7] in order to optimize the design from the hardware point of view and to verify the performances expected from simulation studies. Version by version, the detector was improved both increasing the total size of the calorimeter and adopting new hardware solutions based on what we learned from the previous prototypes. The left panel of Figure 3 shows a picture of the first small-scale prototype, made of 
$\mathrm{CsI}(\mathrm{Tl})$, taken during its preparation for the first beam test. Fourteen frames are visible in the picture, each equipped with a matrix of $3 \times 3$ crystals, $4 \mathrm{~mm}$ apart from each other. As discussed in the previous section, the LYSO crystal would have led to better performances as compared to CsI crystal, even if the collected signal had been reduced by the lower light yield ( $\sim 27$ photons $/ \mathrm{keV})$ and the different peak emission wavelength $(\sim 420 \mathrm{~nm})$. However, in order to demonstrate the operating principle of the CaloCube idea, it is important to build a large prototype able to maximize the shower containment: this reason led to the choice of CsI crystals instead of LYSO crystals.
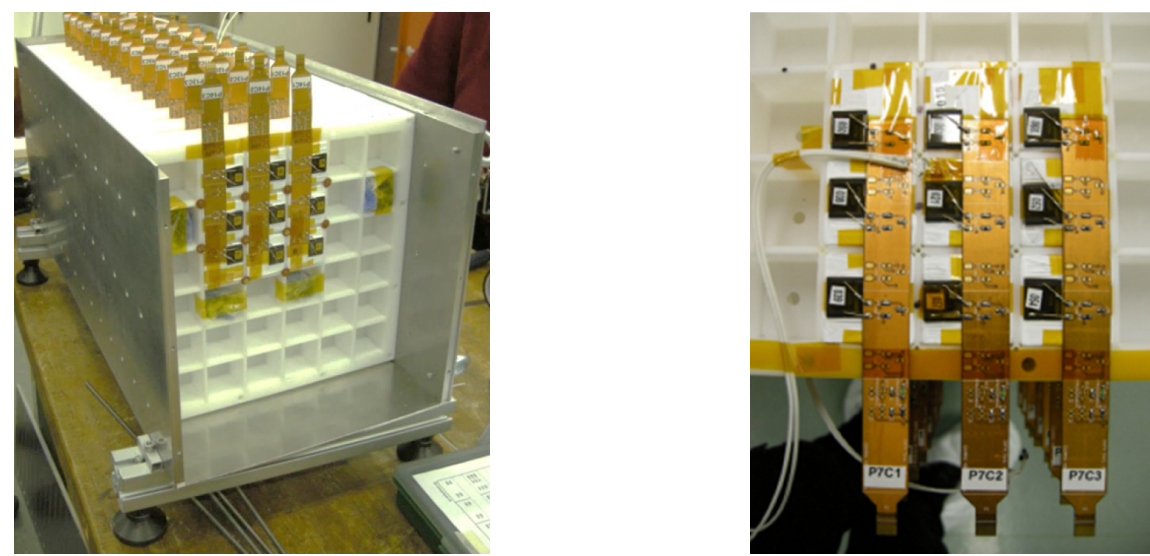

Figure 3. Left panel: The first calorimeter prototype under construction; Right panel: Details of a single layer, showing the nine large area photodiodes placed on the crystals, wrapped in white teflon tape, and the kapton cables used to read out the signals.

The CsI(Tl) has two main advantages. The first one is the fact that its density leads to a good compromise between energy resolution and geometric factor. The second one is that the signal can be easily read using a photodiode, both because of the high light yield ( $\sim 54$ photons $/ \mathrm{keV})$ and because of the peak emission wavelength $(\sim 550 \mathrm{~nm})$, located in a region where the PDs quantum efficiency is large. Moreover, $\mathrm{CsI}(\mathrm{Tl})$ was also chosen for practical reasons: it is widely available on the market at an affordable price. This prototype has a lateral shower containment of about 1.5 Moliere radius and a total depth of 1.35 interaction lengths, corresponding to 28.4 radiation lengths. Signals are readout by means of polyimide flexible printed circuit boards and routed to the front-end board, placed on the side of the calorimeter (see the right panel of Figure 3 for details). The front-end electronics are based on a high dynamic-range, a low-noise Application Specific Integrated Circuit (ASIC), developed by members of the CaloCube collaboration group, and specifically designed for Si-calorimetry in space (CASIS chip [8]). The chosen PD is a large-area $\left(\sim 100 \mathrm{~mm}^{2}\right)$ sensor that, coupled to CsI(Tl) crystals and readout electronics, allows for the clear detection of minimum-ionizing protons with a signal-to-noise ratio of about 15 . One of the most challenging requirements for the instrument is the very large dynamic range needed to detect $\mathrm{PeV}$ protons. According to the simulation, an interacting proton can deposit up to $10 \%$ of its kinetic energy in a single CsI(Tl) crystal of $3.6 \mathrm{~cm}$ size. Considering that non-interacting minimum ionizing protons deposit about $20 \mathrm{MeV}$, the necessary dynamic range is in the order of $10^{7}$. This can be accomplished by also using a second PD of small area $\left(\sim 1 \mathrm{~mm}^{2}\right)$. Many laboratory tests have been performed to compare and characterize various PD responses and to test the system readout in order to optimize the light collection efficiency.

\section{Test with Particle Beams}

The first prototype version was tested at the CERN Super Proton Synchrotron (SPS) with different particle beams. In 2013 and 2015, it was exposed to ion beams (of 12.8 and $30 \mathrm{GeV}$ energy per nucleon) containing $\mathrm{A} / \mathrm{Z}=2$ fragments produced by a primary $(\mathrm{Pb} / \mathrm{Ar}$ ) beam colliding with a target (Be/Poly). The experimental setup also included a Si tracking system, placed in front of the calorimeter, to provide tracking information and $\mathrm{Z}$ tagging. The single-crystal performances were 
studied by selecting non-interacting ions. The responses were equalized by normalizing to the energy deposit of non-interacting He nuclei, the most abundant fragments. The showers developing inside the calorimeter were classified on the basis of the starting point, which can determine the shower containment. A first glance at the performance in a large energy release region can be obtained from Figure 4, which shows in the left panel, the good linearity in the response of the prototype from the low- $\mathrm{Z}\left({ }^{2} \mathrm{H}\right)$ up to the high- $\mathrm{Z}$ ions $(\mathrm{Fe})$, while in the right panel, the correlation observed between the energy released in the first and second layers of the prototype for non-showering particles, pointing out the charge separation power of the calorimeter for the different nuclei.
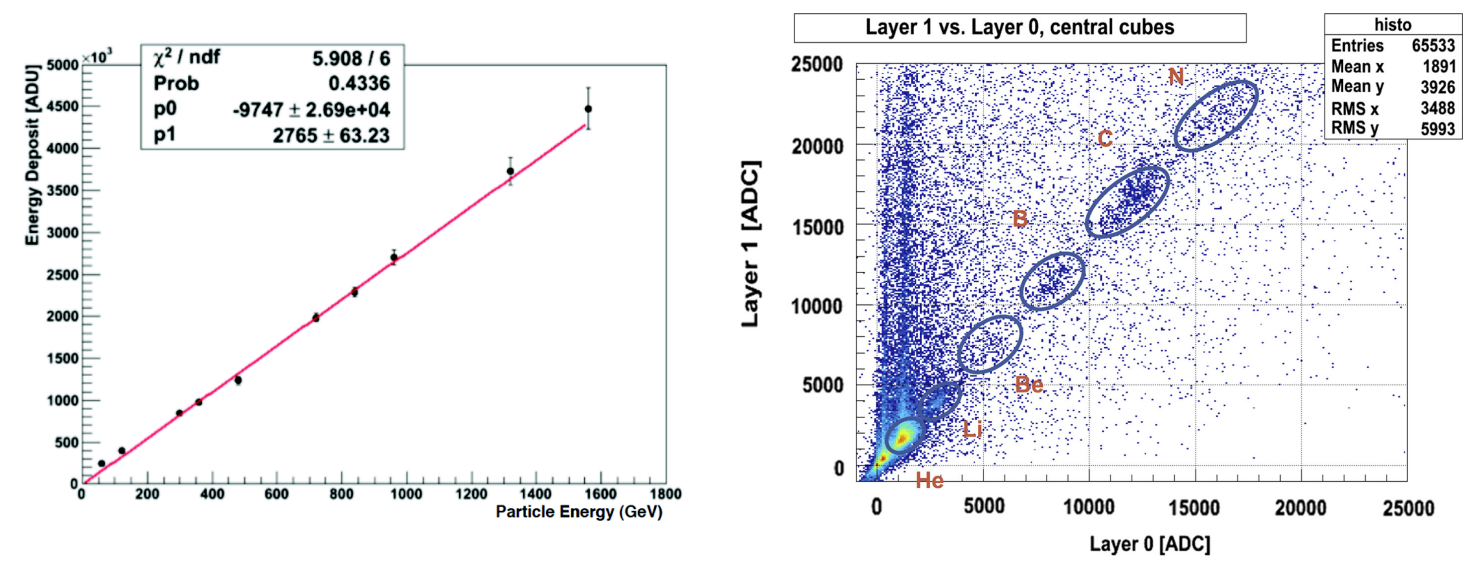

Figure 4. Left panel: Linearity of the response of the prototype to the $30 \mathrm{GeV} / \mathrm{n}$ beam as a function of the energy of the various ions; Right panel: Correlation between energy deposited by non-showering particles in the first and second layer of our prototype.

Moreover the left panel of Figure 5 shows the energy resolution for different ions, at a fixed shower containment. In a first approximation, the shower generated by a nucleus can be described as a superposition of independent showers generated by A nucleons, where A is the mass number. The ions in the beam have the same kinetic energy per nucleon; thus, while the total energy deposit scales as $\mathrm{A}$, the energy resolution scales approximately as $1 / \sqrt{A}$ due to the progressively reduced shower fluctuations. A Fluka-based model of the prototype has been developed including very fine tuning of the simulation, which is necessary to reproduce the beam-test data. In particular, an additional spread of $4.5 \%$ on the single-crystal responses and an optical cross-talk of $14 \%$ were introduced. These effects are consistent with the non-optimized light collection observed in the first version of the prototype and some instrumental issues related to the readout set-up. The predicted response is shown in the left (open symbols) and in the right panel of Figure 5 in comparison with the real data.
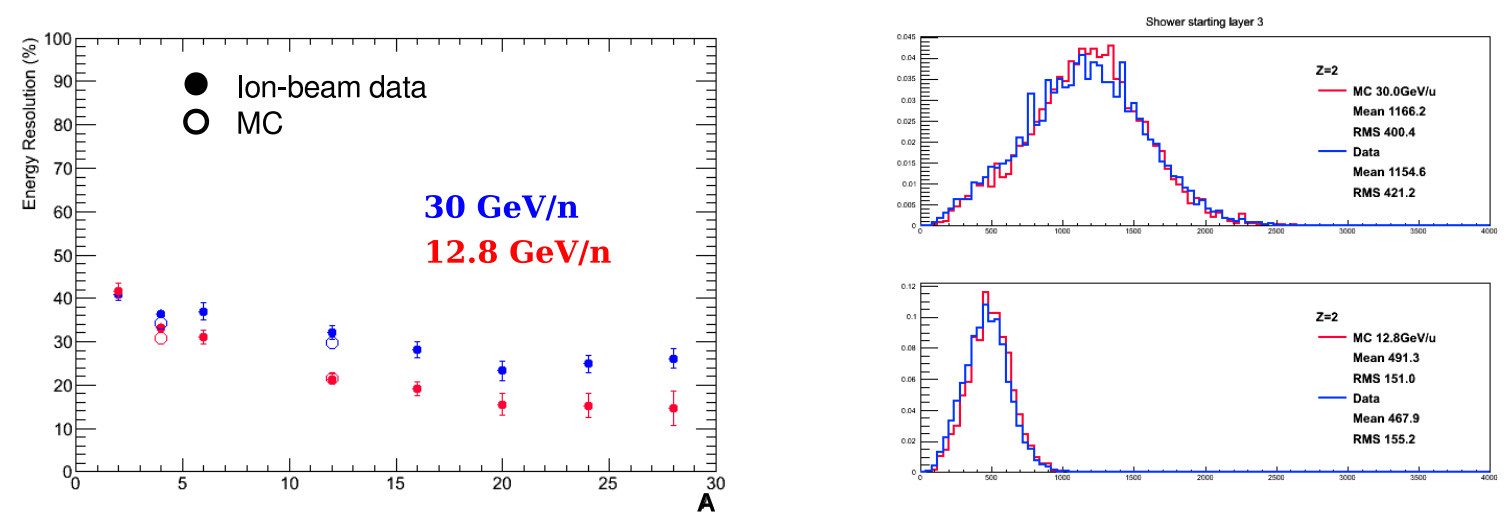

Figure 5. Left panel: Energy resolution as a function of the ion mass number and of the beam energy for showers having the same containment; Right Panel: Distribution of the energy deposit (in MIP units) of $30 \mathrm{GeV} / \mathrm{n}$ (upper panel) and $12.8 \mathrm{GeV} / \mathrm{n}$ (bottom panel) He ions. 
During the beam test at CERN in the summer 2015, the prototype was initially exposed to $\mu$ beams to equalize the response of all the cubes that compose the calorimeter (see the left panel of Figure 6). Then, an estimate of the energy resolution was determined by exposing the calorimeter to electron beams of different energies and determining the total deposited energy. A preliminary result is shown in the right panel of Figure 6 referring to a beam of $50 \mathrm{GeV}$ electrons. The corresponding resolution is at a level of $1.5 \%$, in good agreement with the expected result.
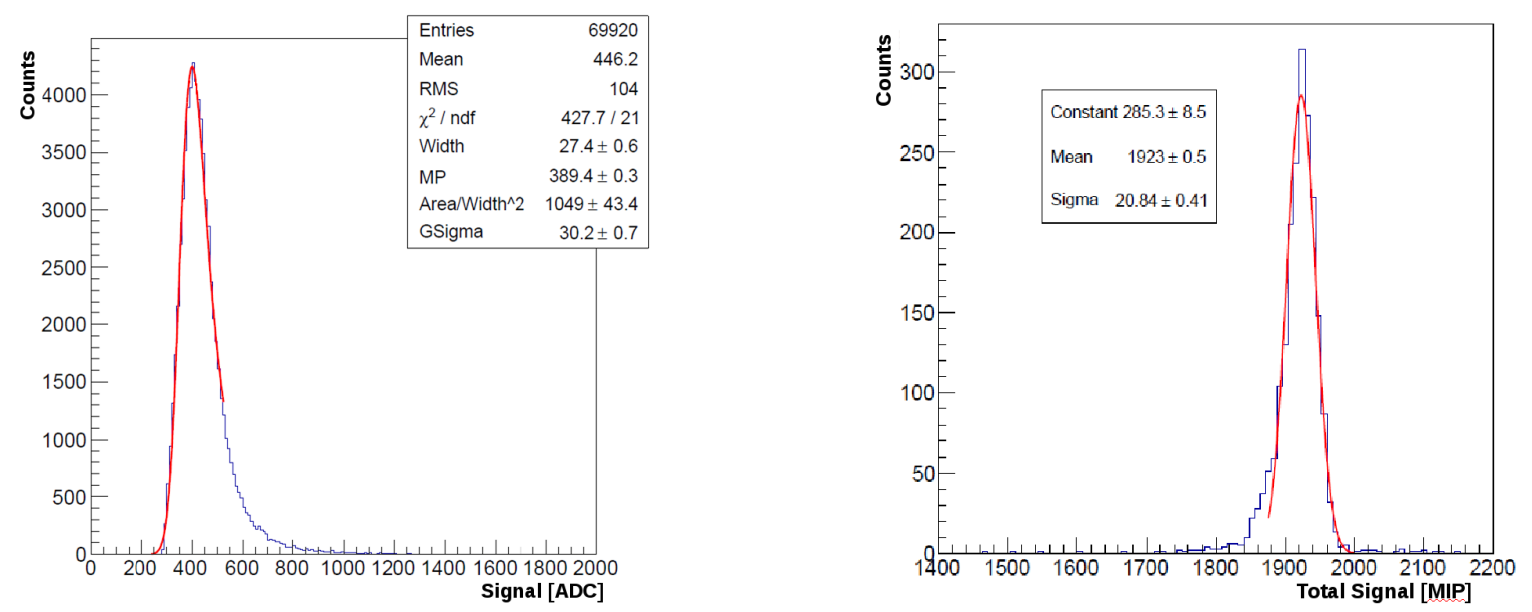

Figure 6. Left Panel: Signal induced by minimum interacting particles (150 GeV muons) in a cube, used to equalize the crystal responses, fitted to the expected Gaussian distribution (red curve); Right Panel: Measured distribution of total energy (expressed in MIP units) released with a $50 \mathrm{GeV}$ electrons beam, fitted to the expected Gaussian distribution (red curve).

\section{The Prototype 2.0}

A new prototype, with completely redesigned mechanics, was assembled in 2016. A schematic view of the latest version is shown in Figure 7. It is made of 18 layers, each layer made of a matrix of $5 \times 5 \mathrm{CsI}(\mathrm{Tl})$ crystals of $3.6 \mathrm{~cm}$ side spaced at $0.3 \mathrm{~cm}$, for a total longitudinal size of $35 X_{0}\left(1.7 \lambda_{I}\right)$ and a lateral size of $2.5 R_{M}$ from the center. Each crystal is wrapped using Vikuiti, a reflective material with a high light collection efficiency: a property that is particularly important to eliminate optical cross talk between adjacent cubes, present in the first prototype (see previous section). As in the first prototype, on each scintillator, two photodiodes are mounted: a large PD (VTH2090 with an active area of $84.6 \mathrm{~mm}^{2}$ ) and a small PD (VTP9421H with an active area of $1.6 \mathrm{~mm}^{2}$ ) that are expected to saturate the electronics in case the energy deposit inside a single crystal is larger than $\sim 25$ and $\sim 2500 \mathrm{GeV}$, respectively. In order to reduce the direct ionization effect, both PDs are mounted sideways with respect to the incoming direction of the test beam and then connected to a kapton cable that brings the signals to the front-end electronics. The front-end electronics are based on the HiDRa chip [8] made up of 28 channels, each one connected to a charge sensitive amplifier followed by a correlated double sampling circuit. The amplifier has two different available gain regimes (a ratio 1:20) and a real-time control feedback network to select the gain, depending on the amplitude of the input signal. The high dynamic range $(52.6 \mathrm{pC})$, the low noise $(2280 \mathrm{e}+7.6 \mathrm{e} / \mathrm{pF})$, and the low consumption $(2.8 \mathrm{~mW} /$ channel) make this chip ideal for space applications. The present version of the prototype was tested making use of different particles beams (muons, electrons, protons, and ions) acquired at the CERN SPS. While the analysis of this data is still ongoing, some preliminary results are discussed in the following sections. The performances of the prototype for electromagnetic showers were investigated using electrons beams between 50 and $280 \mathrm{GeV}$. 

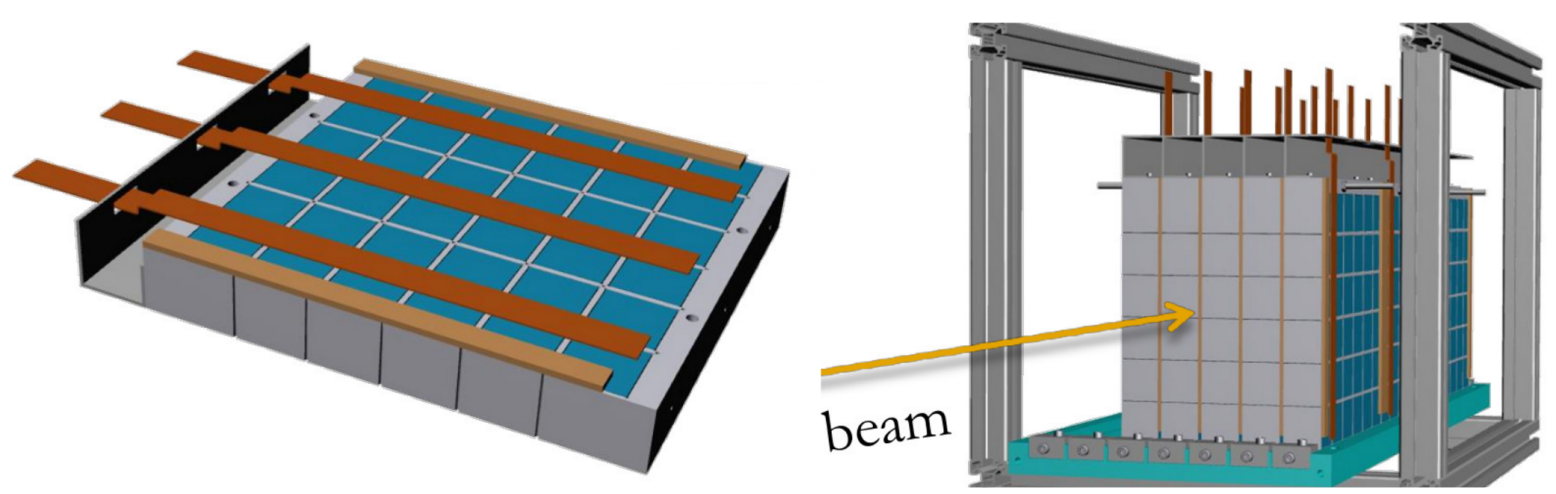

Figure 7. Conceptual design of the latest version of the CaloCube prototype built in 2016: on the left, one of the 18 layers; on the right, the complete detector.

Figure 8 shows the linearity and resolution for the energy, which is reconstructed in three different ways: using large PDs only (blue), using small PDs only (red), and combining the two sets of information (green), so that small PDs are used for the cubes where the large PDs become saturated and large PDs are used in all other cases where small PDs have a low signal. As expected, in the case of the large PDs only, saturation starts to significantly spoils the performances between 150 and $200 \mathrm{GeV}$, whereas, in the case of the small PDs only, $\sigma_{E} / E$ is quite large below this energy because of the small amount of the detected signal. Anyway, the combined results lead to a nonlinearity below $1 \%$ and an energy resolution better than $1.5 \%$ on all parts the energy range. The performances of the prototype for hadronic showers was investigated using proton beams between 100 and $350 \mathrm{GeV}$. Figure 9 shows the total deposit and the energy resolution as a function of the shower starting layer, defined as the first layer having an energy deposit greater than the corresponding $15 \mathrm{MIP}$ signal. As can be seen, when selecting showers which started before the fifth layer, the energy resolution is better than $40 \%$, consistent with the expectations. All the results discussed in this section are better than the ones relative to the previous version of the prototype [9], confirming that the current implementation of the detector satisfies the energy resolution requirements for future space satellite experiments.
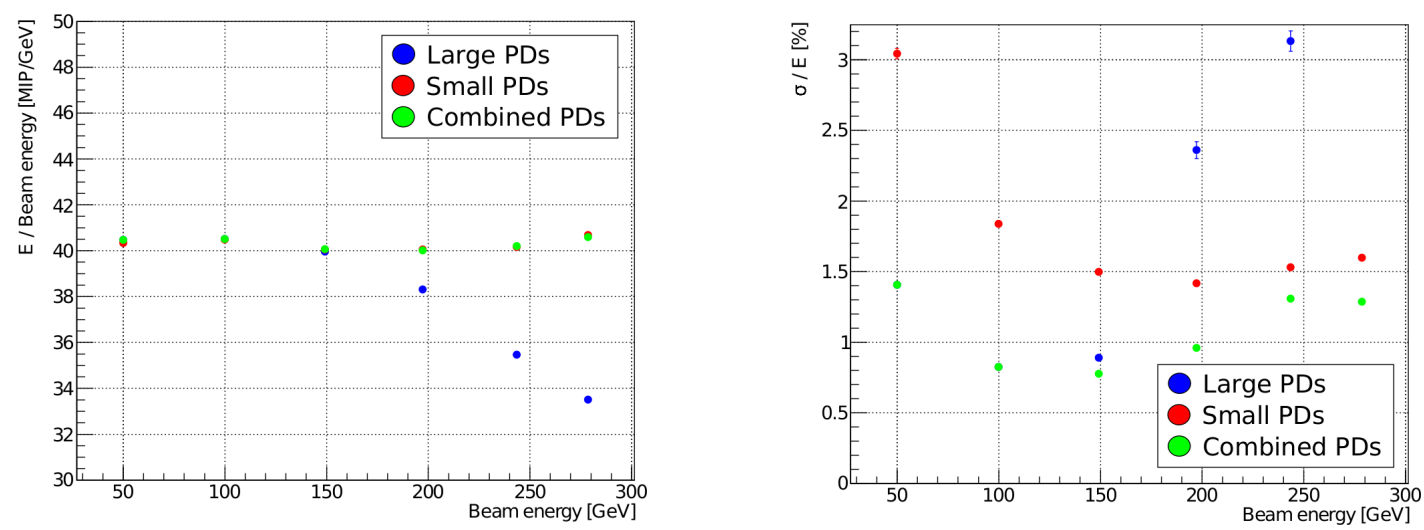

Figure 8. Prototype performances for incident electrons in the energy range between 50 and $280 \mathrm{GeV}$ : Average deposit/beam energy (left) and energy resolution (right). The energy is reconstructed using large photodiodes (PDs) only (blue), small PDs only (red), or by combining the two sets of information (green). 

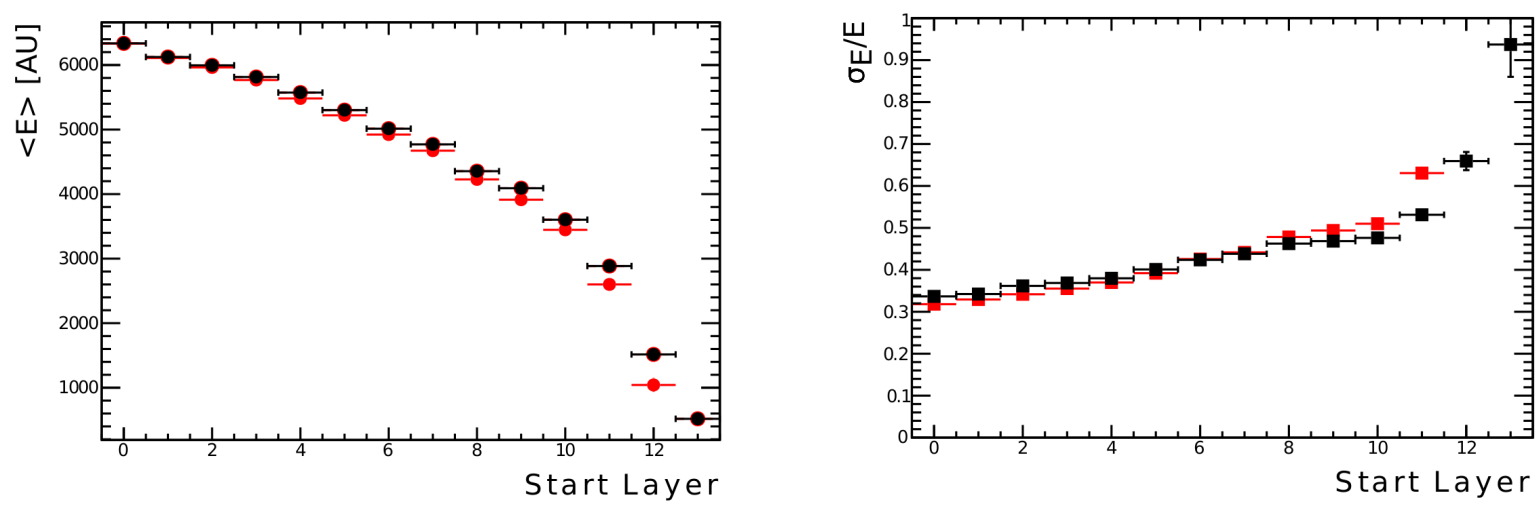

Figure 9. Prototype performances for incident $350 \mathrm{GeV}$ protons: average deposit (left) and energy resolution (right). The mean and the standard deviation of the distributions were both derived from histogram statistics (black) and from Gaussian fit (red).

\section{The Tracker-In-Calorimeter Project}

The CaloCube project is focused on the optimization of a calorimeter for the detection of charged cosmic rays, but, in a general multipurpose experiment in space, the detection of $\gamma$ rays also plays an important role. An important requirement for $\gamma$-rays astronomy is to have a good angular resolution in order to allow precise identification of astrophysical sources in space. This is generally obtained by placing a tracker external to the calorimeter, but, as discussed in the following, this solution decreases the geometric factor of the apparatus. The optimization of the detector, in order to have a good angular resolution needed for $\gamma$ rays, and a good geometric factor needed for charged particles, is the main purpose of the Tracker-In-Calorimeter (TIC) project. TIC is a one-year R\&D project, approved and financed by INFN (Italy) in 2017. In cosmic rays experiments, such as Fermi-LAT [10], the direction of the incident particle is reconstructed using an external tracker made of passive layers (like tungsten) and active position sensitive layers (such as a silicon microstrip detector). In this case, the angle of the $\gamma$ rays is indirectly measured from the tracks of the electron-positron pair formed by conversion in passive layers. The main disadvantage of this configuration is that the acceptance is limited by the large level arm between silicon layers needed to have good track reconstruction performances. In addition, tungsten layers require an additional allocation of a fraction of the mass budget that could instead be used to increase the size of the calorimeter. Passive layers also induce fragmentation of nuclei, thus worsening charge reconstruction performances. All these drawbacks are solved by the TIC approach, where silicon detectors are moved inside the calorimeter, except for a couple of external layers dedicated mainly to charge and track reconstruction of charged particles. This solution exploits the scintillator layers to develop the shower and the silicon detectors to measure the lateral profile. In this case, the angle of the $\gamma$ rays is reconstructed indirectly from the lateral profile of the shower sampled at different depths in the calorimeter, in a similar approach to the one adopted by the LHCf experiment [11].

The two different methods just described are depicted in Figure 10. The development and the optimization of the TIC design are still under study. However, even if the details are not yet defined, preliminary studies show that the idea is promising. Different TIC geometries have been investigated using simulations, leading to an angular resolution in the order of 0.5 for vertical $\gamma$-rays above $10 \mathrm{GeV}$. A prototype has been built inserting several spare silicon layers from the DAMPE experiment inside the latest version of the CaloCube prototype. The detector was tested at the CERN accelerators using electrons with 1-200 GeV incident energy and 0-20 incident angle. The analysis of data is ongoing. 

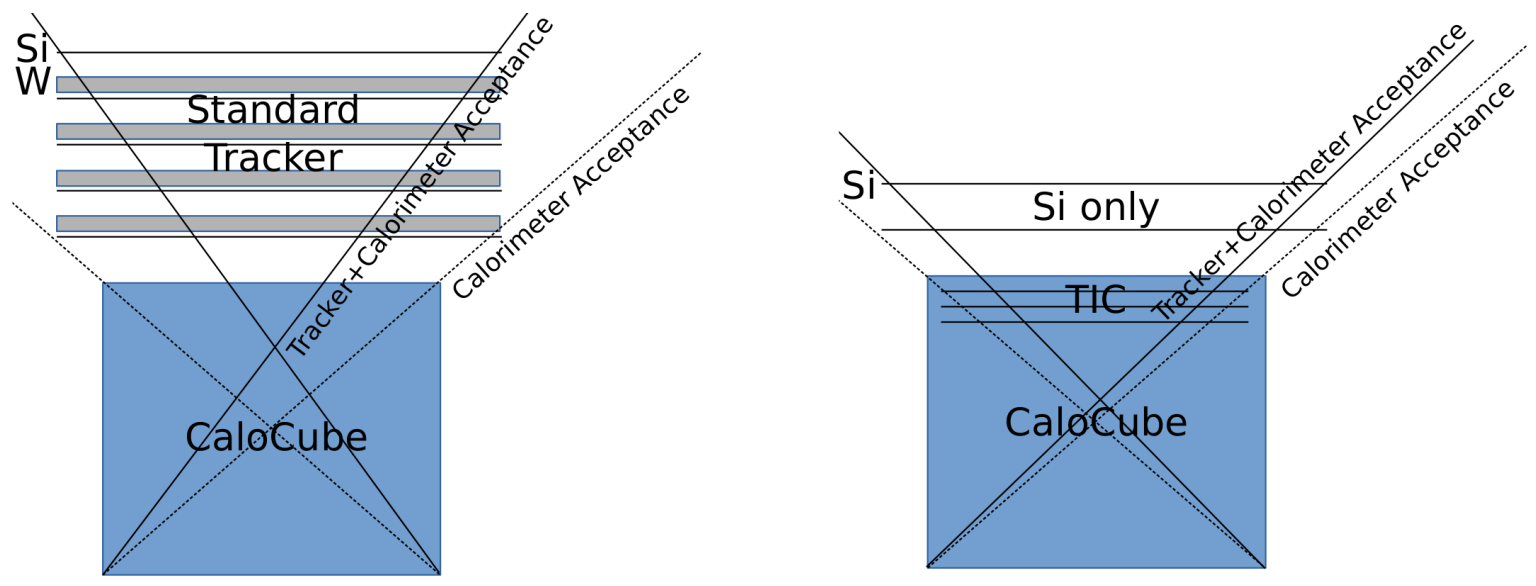

Figure 10. Schematic design of the two different methods for angle reconstruction: On the left, the standard approach of a $\mathrm{Si}+\mathrm{W}$ tracker external to the calorimeter, and on the right, the TIC approach of a $\mathrm{Si}$ tracker inside (and a couple of Si layers outside) the calorimeter.

\section{Conclusions}

In order to extend direct measurements of cosmic ray fluxes of at least one order of magnitude in energy in respect to the maximum value currently achievable, the CaloCube project proposed a cubic calorimeter made of cubic scintillator crystals equipped with photodiodes. Being as homogeneous and isotropic as possible and having five usable faces for particles detection, this design ensures a good particle energy resolution ( $<2 \%$ for electromagnetic showers, $<40 \%$ for hadronic showers) and a good effective geometric factor $\left(>3.5 \mathrm{~m}^{2} \mathrm{sr}\right.$ for electromagnetic showers, $>2.5 \mathrm{~m}^{2} \mathrm{sr}$ for hadronic showers), as confirmed by FLUKA simulations. These performances are consistent with experimental tests on a large prototype made of 18 layers, each layer composed of a $5 \times 5$ matrix of $\mathrm{CsI}(\mathrm{Tl})$ scintillator cubes, each cube equipped with large and small area photodiodes. In order to optimize the detector not only for the detection of charged particles, but also for the detection $\gamma$-rays, a new approach of integrating a silicon tracker inside the calorimeter is being investigated by the TIC project, this being the natural avenue of development for the CaloCube project.

Author Contributions: All the authors contributed equally to this work.

Funding: This work was supported by the Istituto Nazionale di Fisica Nucleare (INFN), through the CaloCube project, and by the H2020 project AIDA-2020, GA no. 654168. The authors thank CERN for the allocation of beam time at the North Area test facility.

Conflicts of Interest: The authors declare no conflict of interest. The funders had no role in the design of the study; in the collection, analyses, or interpretation of data; in the writing of the manuscript, and in the decision to publish the results.

\section{References}

1. Chang, J.; Ambrosi, G.; An, Q.; Asfandiyarov, R.; Azzarello, P.; Bernardini, P.; Bertucci, B.; Cai, M.S.; Caragiulo, M.; Chen, D.Y.; et al. The DArk Matter Particle Explorer mission. Astropart. Phys. 2017, 95, 6-24. [CrossRef]

2. Bongi M.; Adriani, O.; Albergo, S.; Auditore, L.; Bagliesi, M.G.; Berti, E.; Bigongiari, G.; Boezio, M.; Bonechi, L.; Bonechi, S.; et al. CALOCUBE: An approach to high-granularity and homogenous calorimetry for space based detectors. J. Phys. Conf. Ser. 2015, 587, 012029. [CrossRef]

3. D'Alessandro, R.; Adriani, O.; Agnesi, A.; Albergo, S.; Auditore, L.; Basti, A.; Berti, E.; Bigongiari, G.; Bonechi, L.; Bonechi, S.; et al. Calocube - A highly segmented calorimeter for a space based experiment. Nucl. Instrum. Methods Phys. 2016, A824, 609-613. [CrossRef]

4. Ferrari, A.; Sala, P.R.; Fasso, A.; Ranft, J. Fluka: A Multi-Particle Transport Code. Available online: http: / / citeseerx.ist.psu.edu/viewdoc/summary?doi=10.1.1.393.4720 (accessed on 8 February 2019). 
5. Dong, Y.; Xu, M.; Wang, Z.; Adriani, O.; Albergo, S.; Ambrosi, G.; Azzarello, P.; Bai, Y.; Bao, T.; Bernardini, P.; et al. A novel 3-D calorimeter for the High Energy cosmic-Radiation Detection (HERD) Facility onboard China's Future Space Station. Proc. Sci. 2017, 301, 253.

6. Mori, N.; Adriani, O.; Basti, A.; Bigongiari, G.; Bonechi, L.; Bonechi, S.; Bongi, M.; Bottai, S.; Brogi, P.; Alessandro, R.D.; et al. Homogeneous and isotropic calorimetry for space experiments. Nucl. Instrum. Methods Phys. 2013, A732, 311-315. [CrossRef]

7. Vannuccini, E.; Adriani, O.; Agnesi, A.; Albergo, S.; Auditore, L.; Basti, A.; Berti, E.; Bigongiari, G.; Bonechi, L.; Bonechi, S.; et al. CaloCube: A new-concept calorimeter for the detection of high-energy cosmic rays in space. Nucl. Instrum. Methods Phys. 2017, A845, 421-424. [CrossRef]

8. Bonvicini, V.; Orzan, G.; Zampa, G.; Zampa, N. A Double-Gain, Large Dynamic Range Front-end ASIC with A/D Conversion for Silicon Detectors Read-Out. IEEE Trans. Nucl. Sci. 2010, 57, 2963-2970. [CrossRef]

9. Pacini, L.; Adriani, O.; Agnesi, A.; Albergo, S.; Auditore, L.; Basti, A.; Berti, E.; Bigongiari, G.; Bonechi, L.; Bonechi, S.; et al. CaloCube: an innovative homogeneous calorimeter for the next-generation space experiments. J. Phys. Conf. Ser. 2017, 928, 012013. [CrossRef]

10. Atwood, W.B.; Abdo, A.; Ackermann, M.; Althouse, W.; Anderson, B.; Axelsson, M.; Baldini, L.; Ballet, J.; Band, D.L.; Barbiellini, G.; et al. The Large Area Telescope on the Fermi Gamma-Ray Space Telescope Mission. Astrophys. J. 2009, 697, 1071-1102. [CrossRef]

11. Adriani, O.; Bonechi, L.; Bongi, M.; Castellini, G.; D'Alessandro, R.; Faus, D.A.; Fukui, K.; Grandi, M.; Haguenauer, M.; Itow, Y.; et al. The LHCf detector at the CERN Large Hadron Collider. J. Instrum. 2008, 3, S08006.

(C) 2019 by the authors. Licensee MDPI, Basel, Switzerland. This article is an open access article distributed under the terms and conditions of the Creative Commons Attribution (CC BY) license (http:/ / creativecommons.org/licenses/by/4.0/). 